department. This isolates the departments similar to the encapsulated operation of the wards.

To ensure that staff are available, nonessential vacation allowances have been suspended. Because suspended travel may incur financial costs, one of the first items communicated to staff included the Ministry's intention to compensate staff for disruptions to personal travel. This action reduced anxiety and allowed personnel to focus on their duties.

Communication is vital. Regular updates are given to staff to inform them of essential developments, which are communicated via town halls, e-mails, and social media, depending on urgency. Senior management also increased the frequency of their presence in the wards to ensure staff engagement.

Recognition is important. Special compensation was announced for frontline staff. Although the actual reward for going above and beyond the call of duty may not come immediately, it is essential that staff be notified early that they will be recognized for their added effort.

In conclusion, infection prevention and control strategies come with varying degrees of immuration. Deploying a multipronged approach that addresses care and safety of staff and patients while underscoring the sustainability service provision is vital. It is imperative that healthcare organizations respond with overly cautious strategies and that they subsequently monitor the effect of these measures to balance the protection of patients with the tenet of providing the least restrictive service. In matters that are time sensitive, leaders should avoid fatiguing their staff with repeated policy changes because it will lead to complacency. The challenges of prolonged pandemics are felt by everyone, and ultimately, it is important to recognize and validate the contribution of individuals.

Acknowledgments. We would like to thank our international colleagues for sharing with us which of our strategies they found most useful.
Financial support. No financial support was provided relevant to this article.

Conflicts of interest. All authors report no conflicts of interest relevant to this article.

\section{References}

1. Ng Y, Li Z, Chua YX, et al. Evaluation of the effectiveness of surveillance and containment measures for the first 100 patients with COVID-19 in SingaporeJanuary 2-February 29, 2020. Morbid Mortal Wkly Rep 2020;69;307-311.

2. Wong JE, Leo YS, Tan CC. COVID-19 in Singapore-current experience: critical global issues that require attention and action. JAMA 2020 Feb 20 [Epub ahead of print]. doi: 10.1001/jama.2020.2467.

3. Weber DJ, Sickbert-Bennett EE, Vinjé J, et al. Lessons learned from a norovirus outbreak in a locked pediatric inpatient psychiatric unit. Infect Control Hosp Epidemiol 2005;26:841-843.

4. Fukuta Y, Muder RR. Infections in psychiatric facilities, with an emphasis on outbreaks. Infect Control Hosp Epidemiol 2013;34:80-88.

5. Poremski D, Kunjithapatham G, Koh D, Lim XY, Alexander M, Lee C. Lost keys: understanding service providers' impressions of frequent visitors to psychiatric emergency services in Singapore. Psychiatr Serv 2016;68:390-395.

6. Mada P, Saldana D, Castano G, Malus M, Sharon Adley N. Influenza outbreaks with a focus on closed psychiatric units: a review article. Glob J Infect Dis Clin Res 2018;4:1-3.

7. Bergin SM, Periaswamy B, Barkham T, et al. An outbreak of Streptococcus pyogenes in a mental health facility: advantage of well-timed whole-genome sequencing over EMM typing. Infect Control Hosp Epidemiol 2018;39: 852-860.

8. Sim K, Chong PN, Chan YH, Soon W. Severe acute respiratory syndromerelated psychiatric and posttraumatic morbidities and coping responses in medical staff within a primary healthcare setting in Singapore. J Clin Psychiatr 2004;65:1120-1127.

9. National Infection Prevention and Control Committee. The National Infection Prevention and Control Guidelines for Acute Healthcare Facilities. Singapore: Ministry of Health; 2017.

\title{
How we mitigated and contained the COVID-19 outbreak in a hemodialysis center: Lessons and experience
}

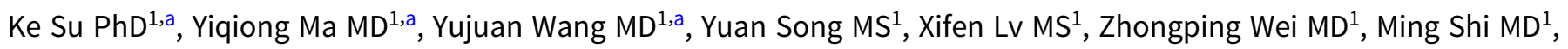 \\ Guohua Ding $M D^{1}$, Bo Shen $M D^{2}$ and Huiming Wang $M D^{1}$ \\ ${ }^{1}$ Department of Nephrology, Renmin Hospital of Wuhan University, Wuhan, Hubei, PR China and ${ }^{2}$ Department of Cardiology, Renmin Hospital of Wuhan \\ University, Wuhan, Hubei, PR China
}

To the Editor-COVID-19 has become a worldwide pandemic. After 2 months of strict control and prevention measures, the COVID-19 epidemic has been contained successfully in Wuhan. We have summarized lessons and experiences related to the reduction of nosocomial COVID-19 in the hemodialysis center for the benefit of healthcare providers and administrations outside China who are facing the challenges of the COVID-19 pandemic.

Author for correspondence: Huiming Wang, E-mail: rm000301@whu.edu.cn.

a Authors of equal contribution.

Cite this article: Su K, et al. (2020). How we mitigated and contained the COVID-19 outbreak in a hemodialysis center: Lessons and experience. Infection Control \& Hospital Epidemiology, 41: 1240-1242, https://doi.org/10.1017/ice.2020.161
Hemodialysis patients are particularly vulnerable to infection and may exhibit greater variations in clinical symptoms and infectivity. Hemodialysis patients are susceptible to infection for the following reasons: (1) Hemodialysis patients require frequent transportation to and from the hospital and their residence to receive dialysis 2 to 3 times per week, which increases the risk of COVID-19 transmission. (2) Hemodialysis patients often require care from family members or caregivers, and if a caregiver is infected, they can transmit the virus to all close contacts, including the hemodialysis patient. (3) The hemodialysis center is a relatively open space with personnel (eg, medical staff and facility workers), patients, and their family members. Thus, many people gather in hemodialysis centers, posing a risk for a virus transmission cluster.

(c) 2020 by The Society for Healthcare Epidemiology of America. All rights reserved. This is an Open Access article, distributed under the terms of the Creative Commons Attribution licence (http://creativecommons.org/licenses/by/4.0/), which permits unrestricted re-use, distribution, and reproduction in any medium, provided the original work is properly cited. 


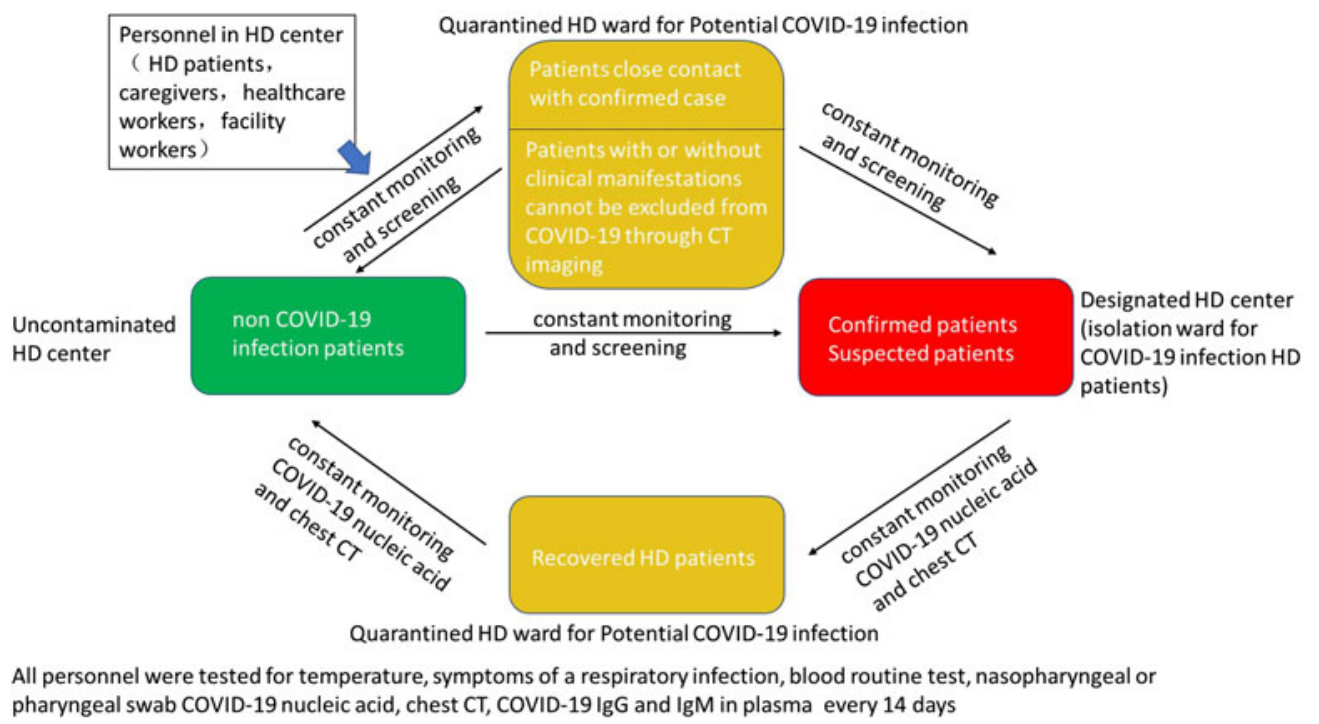

Fig. 1. Personnel cohort in our hemodialysis center and distribution to a different ward.

(4) Hemodialysis patients infected with COVID-19 may lack typical clinical symptoms (eg, fever, cough, or other respiratory symptoms, or the typical ground-glass image computed tomography (CT) scan of the lungs); they may appear asymptomatic or may have mild symptoms. Chest CT images of hemodialysis patients often show acute exudative lesions, lung consolidation, or interstitial changes. These factors increase the difficulty medical workers face in identifying and diagnosing COVID-19 in hemodialysis patients. For these reasons, SARS-CoV-2 spreads quickly in hemodialysis centers.

To prevent the spread of SARS-CoV-2 in our hemodialysis center, the People's Hospital of Wuhan University, we consulted with the Hubei Province public health authorities to develop a series of key strategies to help prevent and mitigate the spread of SARS-CoV-2. Here, we describe the control measures that were implemented.

\section{Repeat and constant screening for infected patients}

To maximize the safety of hemodialysis patients and staff, we continually monitored all persons in our hemodialysis center, including hemodialysis patients and their family members (or caregivers), healthcare workers, and facility workers. We recommend monitoring body temperature and respiratory symptoms, conducting routine blood routine tests, conducting nasopharyngeal or pharyngeal swab SARS-CoV-2 nucleic acid tests, and performing chest CT scans to screening for COVID-19. ${ }^{1}$ Testing for SARS-CoV-2 IgG and IgM antibodies in serum is also recommended. ${ }^{2}$ Chest CT scans are very important in screening hemodialysis patients for COVID-19. We repeated CT scans every 2 weeks to recognize and isolated patients as early as possible in the incubation period.

\section{Evaluation and classification on each person by epidemic situation}

Based on screening results and the Guidelines of the China National Health Commission (6th and 7th editions), personnel in our hemodialysis center can be classified into 5 groups: (1) confirmed cases: a person with laboratory confirmation of COVID-19 infection (COVID-19 nucleic acid testing positive), irrespective of clinical signs and symptoms; (2) suspected cases: patients who satisfy epidemiological and clinical criteria (fever or respiratory symptoms and typical
CT imaging features) but without laboratory confirmation; (3) patients with clinical manifestations but who cannot be excluded from COVID-19 through CT imaging; (4) those who have had close contact with a confirmed case; and (5) non-COVID-19 patients.

\section{Allocation and circulation between designated facilities}

We distributed hemodialysis patients to different hemodialysis centers or hospitals according to the screening results as follows (Fig. 1):

(1) Hemodialysis patients with confirmed or suspected COVID-19 infection were required to be admitted to a negative pressure isolation ward of specified hospitals where only hemodialysis patients with COVID-19 were cared for. If the capacity of the isolation facility was overloaded, the "fixed dialysis care model" outlined below was followed. ${ }^{3}$

(2) Patients who were not SARS-Cov-2 positive continued hemodialysis at the original uncontaminated hemodialysis center.

(3) Hemodialysis patients with clinical manifestations but who could not be excluded from COVID-19 through CT imaging and who had had due to close contact with a confirmed case remained hospitalized in a quarantined ward and received continuous renal replacement therapy (CRRT). Dialysis shifts, dialysis units, and caregiver staff were not be changed to prevent cross contamination and infection. Contact with relatives was minimized. These quarantine hemodialysis patients underwent the testing outlined previously during the 14-day quarantine period. Once a hemodialysis patient converted to a confirmed case, the patient was treated under confirmed case management protocols. Screening for patients only once was not enough; repeated screening was needed to identify probable cases.

(4) When hemodialysis patients with COVID-19 recovered, they were transferred to a quarantine ward for recovered patients for 14 days of observation. After 2 negative nucleic acid tests, the patient could be transferred to the uncontaminated hemodialysis center. If any healthcare personnel were confirmed with COVID-19 or had a probable case, they were also quarantined. ${ }^{4}$

These measures have proven effective. After the beginning of outbreak, there were 37 COVID-19 cases among 230 hemodialysis 
patients (16.09\%) and 4 cases among 33 staff (12.12\%) who were suspected cases. Furthermore, 7 confirmed and suspected hemodialysis patients died between February 4 and February 13, 2020. ${ }^{5}$ Also, 5 confirmed patients and no healthcare workers or facility workers were infected between February 14 and March 1, 2020. Collectively, these strategies can effectively minimize clusters of infection while providing timely treatment for hemodialysis patients.

\section{Acknowledgments.}

Financial support. No financial support was provided relevant to this article.

Conflicts of interest. All authors report no conflicts of interest relevant to this article.

\section{References}

1. The National Health Commission of PRC. Guideline for diagnosis and treatment of novel coronavirus disease (version 6). February 18, 2020. http://www. nhc.gov.cn/yzygj/s7653p/202002/8334a8326dd94d329df351d7da8aefc2.shtml. Updated February 18, 2020. Accessed February 19, 2020.

2. Guideline for diagnosis and treatment of novel coronavirus disease (version 7). The National Health Commission of PRC website. http://en.nhc.gov.cn/202003/29/c_78469.htm. Updated March 29, 2020. Accessed April 22, 2020.

3. Naicker S, Yang CW, Hwang SJ, Liu BC, Chen JH, Jha V. The novel coronavirus 2019 epidemic and kidneys. Kidney Int 2020. doi: 10.1016/j.kint.2020. 03.001 .

4. Interim additional guidance for infection prevention and control recommendations for patients with suspected or confirmed COVID-19 in outpatient hemodialysis facilities. Centers for Disease Control and Prevention website. https://www.cdc.gov/coronavirus/2019-ncov/healthcare-facilities/dialysis.html. Updates April 12, 2020. Accessed April 22, 2020.

5. Ma Y. 2019 novel coronavirus disease in hemodialysis (HD) patients: report from one HD center in Wuhan, China. medRxiv 2020. doi: 10.1101/2020.02. 24.20027201.

\title{
COVID-19 in the endoscopy ward: A potential risk for gastroenterologists
}

\author{
Ahmad Hormati MD¹, Mohammad Hadi Karbalaie Niya $\mathrm{PhD}^{2}$, Mohammadreza Ghadir MD ${ }^{1}$, Kamran Bagheri Lankarani MD \\ Hossein Ajdarkosh MD², Fahimeh Safarnezhad Tameshkel PhD candidate ${ }^{2}$ and Farhad Zamani MD ${ }^{2}$ (1) \\ ${ }^{1}$ Gastroenterology and Hepatology Disease Research Center, Qom University of Medical Sciences, Qom, Iran, ${ }^{2}$ Gastrointestinal and Liver Diseases Research \\ Center, Iran University of Medical Sciences, Tehran, Iran and ${ }^{3}$ Health Policy Research Center, Shiraz University of Medical Sciences, Fars, Iran
}

To the Editor-COVID-19, an emerging coronavirus disease, is major health problem. As of April 15, 2020, it involved 2,035,299 cases globally, of whom 130,712 died. ${ }^{1,2}$ Coronaviruses comprise a range of positive-sense RNA viruses including several zoonotic viruses. Severe acute respiratory coronavirus type 2 (SARS-CoV-2) has become serious and devastating threat worldwide; it spreads quickly among humans via 2 major routes: respiratory and fecal-oral. Contaminated droplets are the major source of the virus transmission, and the disease initially occurs in the respiratory tract. ${ }^{3}$ Human-to-human transmission by direct contact at $<1 \mathrm{~m}$ is the most effective way to transfer an infective amount of the virus, and this type of contact occurs frequently in medical centers during routine practice. In addition, the stability of the virus on solid surfaces is high, which puts the medical center setting at high risk for viral contamination. ${ }^{4}$ Thus, medical centers are critical areas for disease control. Unrecognized COVID-19 cases referred for routine endoscopic practice are a probable source of viral contamination of facilities. Endoscopic procedures can facilitate airborne transmission as well as contamination of surfaces. Medical personnel are at risk for disease dissemination by various routes. ${ }^{5}$ Iran, a Middle Eastern country, has a high rate of COVID-19. In Iran, 76,389 confirmed cases and 4,777 deaths have been reported, and Iran ranks first among Middle Eastern countries and eighth in the world for COVID-19 prevalence. ${ }^{1,2}$ Here,

Author for correspondence: Professor Farhad Zamani, E-mail: zamani.f@iums.ac.ir Cite this article: Hormati A, et al. (2020). COVID-19 in the endoscopy ward: A potential risk for gastroenterologists. Infection Control \& Hospital Epidemiology, 41: 1242-1243, https://doi.org/10.1017/ice.2020.160 we report the COVID-19 prevalence in Iranian endoscopic wards among gastroenterologists.

All gastroenterologists who are on call and who routinely worked in referral hospitals are at risk of viral infection, and unusual presentation of COVID-19 may expose them unexpectedly to the disease. ${ }^{6,7}$ We surveyed these specialists to evaluate the prevalence of COVID-19 and hospitalization using a standard protocol for COVID-19 diagnosis. We distributed a questionnaire to all gastrointestinal wards in Iran, and we received responses from $\sim 480$ gastroenterologists by March 26,2020 . Our data analysis revealed that 51 of these gastroenterologists (10.6\%) had COVID-19 symptoms that had been confirmed by a laboratory test. Among these cases, $60 \%$ had moderate disease, $30 \%$ had mild disease, and $10 \%$ had severe disease. Furthermore, patients with gastrointestinal symptoms may represent a source of underdiagnosed COVID-19 cases because they do not present with classic symptoms of the disease such as fever and respiratory signs. ${ }^{6,7}$

Personal protection equipment (PPE) is crucial for preventing exposure to the virus. Various protocols have been recommended for PPE for clinicians as well as patients. Endoscopy patients should wear a special gown, mask, and gloves based on their classification as intermediate- or high-risk patients. ${ }^{5}$ Endoscopy personnel should use standard protective clothing and should maintain a reasonable distance of contact with all patients, especially suspicious cases. The minimal personal protective equipment (PPE) recommended elsewhere should be used, especially by at-risk individuals such as gastroenterologists (Fig. 1). ${ }^{5}$

In conclusion, gastroenterologists, as on-call experts, are at higher risk for COVID-19 via the fecal-oral route as well as the

(c) 2020 by The Society for Healthcare Epidemiology of America. All rights reserved. This is an Open Access article, distributed under the terms of the Creative Commons Attribution licence (http://creativecommons.org/licenses/by/4.0/), which permits unrestricted re-use, distribution, and reproduction in any medium, provided the original work is properly cited. 